\title{
Magnetically Driven Warping and Precession of Accretion Disks: Implications for Exotic Stellar Variabilities
}

\author{
Dong Lai \\ Department of Astronomy, Cornell University, Ithaca, NY 14853, USA
}

\begin{abstract}
The inner region of the accretion disk around a magnetized star is subjected to magnetic torques that induce warping and precession of the disk. These torques arise from interactions between the stellar field and the induced electric currents in the disk. These novel magnetic effects give rise to some "exotic" stellar variabilities, and may play an important role in explaining a number of puzzling behaviors related to disk accretion onto magnetic stars, such as $\mathrm{mHz}$ QPOs in X-ray pulsars, long-term periodicities of X-ray binaries (including precession of jets), low-Frequency $(10-50 \mathrm{~Hz})$ QPO's in low-mass X-ray binaries, and photometric variabilities of $\mathrm{T}$ Tauri stars.
\end{abstract}

\section{Introduction}

Disk accretion onto a magnetic star occurs in a variety of astrophysical contexts, including accreting neutron stars, white dwarfs and pre-main-sequence stars (e.g., Frank et al. 1992). The basic picture of disk-magnetosphere interaction is well-known: at large radii the disk is unaffected by the stellar magnetic field; a somewhat sudden transition occurs when the stellar field disrupts the disk at the magnetospheric boundary, and channels the plasma onto the polar caps of the star. The magnetosphere boundary is located where the magnetic and plasma stresses balance,

$$
r_{m}=\eta \mu^{4 / 7}\left(G M \dot{M}^{2}\right)^{-1 / 7},
$$

where $M$ and $\mu$ are the mass and magnetic moment of the central star, $\dot{M}$ is the mass accretion rate, $\eta$ is a dimensionless constant of order unity.

Because of its intrinsic importance for a wide range of astrophysical systems, a large number of theoretical papers have been written on the subject of the interaction between accretion disks and magnetized stars (see references in Lai 1999 and in Shirakawa \& Lai 2002a,b), and numerical study of this problem is still in its infancy. Outstanding issues remain, including the efficiency of field dissipation in/outside the disk, whether the disk excludes the stellar field by diamagnetic currents or the field can penetrate a large fraction of the disk, whether the threaded field remains closed (connecting the star and the disk) or becomes open by differential shearing, and whether/how magnetically driven wind is launched from the disk or the magnetosphere/corotation boundary.

Many previous theoretical papers have, for simplicity, adopted the idealized geometry in which the magnetic axis, the spin axis and the disk angular momentum are aligned. However, in Lai (1999), it was shown that under quite general 
conditions, the stellar magnetic field can induce warping in the inner disk and make the disk precess around the spin axis (see §2). Such magnetically driven warping and precession open up new possibilities for the dynamical behaviors of disk accretion onto magnetic stars, and may explain some of the observed variabilities in different stars (including compact objects).

\section{Magnetically Driven Warping/Precession}

Lai (1999) shows that the inner region of the accretion disk onto a rotating magnetized central star is subjected to magnetic torques which induce warping and precession of the disk. The origin of these torques lies in These magnetic torques result from the interactions between the accretion disk and the stellar magnetic field. Depending on how the disk responds to the stellar field, two different kinds of torque arise: (i) If the vertical stellar magnetic field $B_{z}$ penetrates the disk, it gets twisted by the disk rotation to produce an azimuthal field $\Delta B_{\phi}=\mp \zeta B_{z}$ that has different signs above and below the disk $(\zeta$ is the azimuthal pitch of the field line and depends on the dissipation in the disk), and a radial surface current $K_{r}$ results. The interaction between $K_{r}$ and the stellar $B_{\phi}$ gives rise to a vertical force. While the mean force (averaging over the azimuthal direction) is zero, the uneven distribution of the force induces a net warping torque which tends to misalign the angular momentum of the disk with the stellar spin axis. (ii) If the disk does not allow the vertical stellar field (e.g., the rapidly varying component of $B_{z}$ due to stellar rotation) to penetrate, an azimuthal screening current $K_{\phi}$ will be induced on the disk. This $K_{\phi}$ interacts with the radial magnetic field $B_{r}$ and produces a vertical force. The resulting precessional torque tends to drive the disk into retrograde precession around the stellar spin axis.

In general, both the magnetic warping torque and the precessional torque are present. For small disk tilt angle $\beta$ (the angle between the disk normal and the spin axis), the precession angular frequency and warping rate at radius $r$ are given by

$$
\Omega_{p}(r)=\frac{\mu^{2}}{\pi^{2} r^{7} \Omega(r) \Sigma(r) D(r)} F(\theta), \quad \Gamma_{w}(r)=\frac{\zeta \mu^{2}}{4 \pi r^{7} \Omega(r) \Sigma(r)} \cos ^{2} \theta
$$

where $\mu$ is the stellar magnetic dipole moment, $\theta$ is the angle between the magnetic dipole axis and the spin axis, $\Omega(r)$ is the orbital angular frequency, and $\Sigma(r)$ is the surface density of the disk. [Note that the stellar spin frequency $\Omega_{s}$ does not appear in eqs. (2) \& (3) since the variation of the field geometry due to the spin has been averaged out; this is justified because $\left.\Omega_{s} \gg\left|\Omega_{p}\right|,\left|\Gamma_{w}\right|.\right]$ The dimensionless function $D(r)$ is given by

$$
D(r)=\max \left(\sqrt{r^{2} / r_{\mathrm{in}}^{2}-1}, \sqrt{2 H(r) / r_{\mathrm{in}}}\right),
$$

where $H(r)$ is the half-thickness and $r_{\text {in }}$ is the inner radius of the disk. The function $F(\theta)$ depends on the dielectric property of the disk. We can write

$$
F(\theta)=2 f \cos ^{2} \theta-\sin ^{2} \theta
$$


so that $F(\theta)=-\sin ^{2} \theta$ if only the spin-variable vertical field is screened out by the disk $(f=0)$, and $F(\theta)=3 \cos ^{2} \theta-1$ if all vertical field is screened out $(f=1)$. In reality, $f$ lies between 0 and 1 . For concreteness, we shall set $F(\theta)=-\sin ^{2} \theta$ in the following.

We also note the effect of magnetically driven resonances. For a general magnetic field-disk geometry, the vertical magnetic force on a disk element varies with the stellar rotation period. This gives rise to a number of vertical resonances in the disk. Similarly. there exist epicyclic resonances due to the time-dependent radial magnetic force. Although the force expressions are model-dependent, the existence of the resonances appears to be inevitable. These magnetically driven resonances are somewhat similar to the corotation resonance and Lindblad resonances in gravitational systems. The resonances may act as an extra source (in addition to the non-resonant precessional and warping torques discussed above) for generating bending waves and spiral waves in the disk. Near the resonances, fluid elements undergo large out-of-plane and radial excursions, which may lead to thickening of the disk. This may be analogous to the Lorentz resonances (which occur when charged particles move around a rotating magnetic field) in the Jovian ring (e.g., Schaffer \& Burns 1992). However, because of the fluid nature of the disk, the resonances may not lead to sharp edges in the disk.

\section{Dynamics of Warped Disks, Effects of Viscosity, Global Warping Modes and Nonlinear Evolution}

\subsection{Effects of Viscosity}

Since the magnetic torque drives the disk tilt, while the viscosity reduces the tilt, one can derive the criterion for the warping instability. Roughly speaking, the disk warp can grow if the timescale associated with the warping torque is shorter than the viscous time $r^{2} / \nu_{2}$ [where $\nu_{2}$ is the viscosity (measuring the $r$ - $z$ stress) associated with reducing disk tilt]. Since the warping torque is a steep function of $r$, the warping instability occurs only inside a critical radius $r_{w}$. Our analysis (Lai 1999) shows that local warping torque can overcome viscous damping when

$$
\Gamma_{w}>2 \pi^{2} \frac{\nu_{2}}{r^{2}} \Longleftrightarrow \text { Instability. }
$$

Assuming that $\nu_{2} / \nu_{1}$ is independent of $r$, the above equation reduces to

$$
r<r_{w}=\left(\frac{3 \zeta \cos ^{2} \theta}{8 \pi^{2} \mathcal{J}} \frac{\nu_{1}}{\nu_{2}}\right)^{2 / 7}\left(\frac{\mu^{4}}{G M \dot{M}^{2}}\right)^{1 / 7},
$$

where we have used $\Sigma=\left(\dot{M} / 3 \pi \nu_{1}\right) \mathcal{J}$, and $\mathcal{J}$ is a dimensionless function of $r$ which approaches unity in the region far from the inner edge of the disk. Thus $r_{w}$ is typically a few times the canonical Alfúen radius (the magnetosphere boundary). Therefore, as the disk approaches the magnetosphere, its normal vector $\hat{l}$ will tend to be tilted with respect to the stellar spin even if at large radii it is aligned with the spin axis.

Another aspect of the viscous effect is what we call "Magnetic BardeenPetterson Effect". Because of the magnetic precessional torque, the tilted disk 
will be driven into differential precession (with the precession rate dependent on $r$ ). By analogy with the well-known Bardeen-Petterson effect (i.e., the inner region of an accretion disk undergoing Lense-Thirring precession around a rotating black hole tends to align itself with the equatorial plane of the black hole; see Bardeen \& Petterson 1975), we expect that the magnetically driven precession also tends to damp the tilt of the inner disk through the action of viscosity. Setting $\Omega_{p}$ equal to $\nu_{2} / r^{2}$, we obtain the magnetic Bardeen-Petterson radius:

$$
r_{\mathrm{MBP}}=\left(\frac{3 \sin ^{2} \theta}{\pi \mathcal{J} D} \frac{\nu_{1}}{\nu_{2}}\right)^{2 / 7}\left(\frac{\mu^{4}}{G M \dot{M}^{2}}\right)^{1 / 7} .
$$

Inside $r_{\mathrm{MBP}}$, the combined effect of viscosity and precession tends to align the disk normal with the spin axis. We see that typically $r_{\mathrm{MBP}}$ is of the same order as $r_{w}$ (the warping radius) and $r_{m}$ (the magnetosphere radius). Thus the precessional torque has an opposite effect on the disk tilt as the warping torque. However, because of the broad warp-alignment transition expected for the magnetic Bardeen-Petterson effect and the long timescale involved, we expect that the precession-induced alignment will be overwhelmed by the warping instability.

\subsection{Global Warping Modes and Nonlinear Evolution}

Since the precession rate $\Omega_{p}(r)$ depends strongly on $r$, coupling between different rings is needed to produce a global coherent precession. The coupling can be achieved either by viscous stress or through bending waves (e.g., Papaloizou\& Pringle 1983; Papaloizou \& Terquem 1995). In the viscosity dominated regime (i.e., the dimensionless viscosity parameter $\alpha$ greater than $H / r$ ), the dynamics of the warps can be studied using the formalism of Papaloizou \& Pringle (1983) (see also Ogilvie 1999; Ogilvie \& Dubus 2001). We model the disk as a collection of rings which interact with each other via viscous stresses. Each ring at radius $r$ has the unit normal vector $\hat{\mathbf{l}}(r, t)$. In the Cartesian coordinates, with the $z$ axis along the neutron star spin, we write $\hat{\mathrm{l}}=(\sin \beta \cos \gamma, \sin \beta \sin \gamma, \cos \beta)$, with $\beta(r, t)$ the tilt angle and $\gamma(r, t)$ the twist angle. For $\beta \ll 1$, the dynamical warp equation for î (Lai 1999; see Papaloizou \& Pringle 1983; Pringle 1992) reduces to an equation for $W(r, t) \equiv \beta(r, t) e^{i \gamma(r, t)}$ :

$$
\frac{\partial W}{\partial t}-\left[\frac{3 \nu_{2}}{4 r}\left(1+\frac{2 r \mathcal{J}^{\prime}}{3 \mathcal{J}}\right)+\frac{3 \nu_{1}}{2 r}\left(\mathcal{J}^{-1}-1\right)\right] \frac{\partial W}{\partial r}=\frac{1}{2} \nu_{2} \frac{\partial^{2} W}{\partial r^{2}}+i \Omega_{p} W+\Gamma_{w} W
$$

where $\mathcal{J}^{\prime}=d \mathcal{J} / d r$ (we assume that the ratio of $\nu_{2}$ to $\nu_{1}$ is constant). In deriving the above equation, we have used the relations for the radial velocity and surface density: $v_{r}=-3 \nu_{1} \mathcal{J}^{-1} / 2 r$ and $\Sigma=\dot{M} \mathcal{J} / 3 \pi \nu_{1}$. The values and functional forms of $\nu_{1}, \nu_{2}, \Omega_{p}, \Gamma_{w}$ and the dimensionless function $\mathcal{J}(r)$ depend on disk models (see Shirakawa \& Lai 2002a,b for details).

Shirakawa \& Lai (2002) carried out a global analysis of warping/precession modes in a viscous accretion disk, and show that under a wide range of conditions, the magnetic warping torque can overcome viscous damping and make the mode grow. The warping/precession modes are concentrated near the inner edge of the disk (at the magnetosphere-disk boundary), and can give rise to variabilities or quasi-periodic oscillations (QPOs) in the X-ray/UV/optical fluxes from 
X-ray pulsars (see §4). Pfeiffer \& Lai (2002) studied the nonlinear evolution of the warping-precession modes, and found that the mode tends to saturate at a large amplitude (its value depends on the parameters of the system). The implications of such nonlinear behavior remain to be understood.

\section{Applications}

The magnetically driven warping instability and precession help explaining a number of observational puzzles related to stellar variabilities (for more details, see Lai 1999; Shirakawa \& Lai 2002a, 2002b; Pfeiffer \& Lai 2002).

(i) Milli-Hertz QPO's in Accreting X-ray Pulsars: Quasi-Periodic Oscillations (QPO's) with frequencies $1-100 \mathrm{mHz}$ have been detected in at least 11 accreting X-ray pulsars. These $\mathrm{mHz}$ QPOs are often interpreted in terms of the beat frequency model (BFM), in which the observed QPO frequency represents the beat between the Keplerian frequency $\nu_{K}$ at the inner disk radius and the NS spin frequency $\nu_{s}$, or in terms of the Keplerian frequency model (KFM), in which the QPOs arise from the modulation of the X-rays by some inhomogeneities in the inner disk at the Keplerian frequency. However for several sources, more than one QPOs have been detected and the difference in the QPO frequencies is not equal to the spin frequency. Thus KFM and/or the BFM cannot be the whole story. Also note that in both the KFM and the BFM, it is always postulated that the inner disk contain some blobs or inhomogeneities, whose physical origin is unclear. In Shirakawa \& Lai (2002) we suggest a "Magnetic Disk Precession Model" for the $\mathrm{mHz}$ variabilities and QPOs of accreting $\mathrm{X}$-ray pulsars. The magnetically driven precession of the warped inner disk (outside but close to the magnetosphere boundary) can modulate X-ray/UV/optical flux in several ways. We identify $\nu_{\mathrm{QPO}}$ with the global precession frequency driven by the magnetic torques. Our calculations show that under a wide range of conditions, the warping/precession mode is concentrated near the disk inner edge, and the global mode frequency is equal to $A=0.3-0.85$ (depending on details of the disk structure) times the magnetically driven precession frequency at $r_{\text {in }}=r_{m}$. An examination of the observed properties of $\mathrm{mHz}$ QPOs in several systems (such as $4 \mathrm{U} 4 \mathrm{U}$ 1626-67) suggests that some hitherto unexplained QPOs are likely to be results of magnetically driven disk warping/precession.

(ii) Spin evolution of accreting X-ray pulsars: Recent long-term, continuous monitoring of X-ray pulsars with the BATSE instrument on the Compton Gamma Ray Observatory (CGRO) has revealed a number of puzzling behaviors of the spins of these objects (see Bildsten et al. 1997 and references therein). Several well-measured disk-fed systems (e.g., Cen X-3, GX 1+4 and $4 \mathrm{U}$ 1626-67) display sudden transitions between episodes of steady spin-up and spin-down, with the absolute values of spin torques approximately equal (to within a factor of a few). The transition timescale ranges from days to years. It is likely that the magnetically driven disk warping may be a crucial ingredient in determining the spin behaviors of accreting X-ray pulsars. With the magnetic warping torque, the perpendicular state is an "attractor." The observed sign switching of $\dot{\omega}_{s}$ (spin derivative) in several X-ray pulsars may be associated with the "wandering" of the inner disk around this "attractor." Rough estimate based on the magnetic torque indicates that the switching timescale (which depends 
on the stellar field strength, the disk parameters and geometry) ranges from days to years, in agreement with observations.

(iii) Quasi-periodic oscillations in low-mass X-ray binaries: Rapid variability in low-mass X-ray binaries, with weakly magnetized $\left(B \sim 10^{8} \mathrm{G}\right)$ neutron stars, has been studied since the discovery of the so-called horizontal-branch oscillations (HBOs) (see van der Klis 1998). The HBOs are quasi-periodic oscillations (QPOs) (with $Q$-value $\nu / \Delta \nu$ of order a few) which manifest as broad Lorentzian peaks in the X-ray power spectra with centroid frequencies in the range of $15-60 \mathrm{~Hz}$ which are positively correlated with the inferred mass accretion rate. Stella and Vietri (1998) suggested that HBOs (and other low-frequency QPOs) are associated with Lense-Thirring precession of the inner accretion disk around the rotating NS. For this interpretation to be viable, the inner disk must be tilted with respect to the stellar spin axis. Since the Bardeen-Petterson effect tends to keep the inner region of the disk (typically within 100-1000 Schwarzschild radii) co-planar with the star and radiation-driven warping is only effective at large disk radii (Pringle 1996), another mechanism to drive warping in the inner disk is needed. The magnetic warping torque provides a natural source for inducing disk tilt. Moreover, the magnetically driven (retrograde) precession rate is not negligible compared to the Lense-Thirring precession rate, and will contribute to the total precession (Shirakawa \& Lai 2002a).

Other possible applications include (iv) long-term (super-orbital) variabilities in X-ray binaries (including precession of jets); (v) photometric period variations of $T$ Tauri stars.

Acknowledgments. I thank Akiko Shirakawa and Harald Pfeiffer for their important contributions. This work has been supported in part by NSF AST 9986740 and NASA NAG 5-8484, and by the Alfred P. Sloan foundation.

\section{References}

Bardeen, J. M., \& Petterson, J. A. 1975, ApJ, 195, L65

Bildsten, L., et al. 1997, ApJS, 113, 367

Frank, J., King, A., \& Raine, D. 1992, Accretion Power in Astrophysics (Cambridge: Cambridge Univ. Press)

Lai, D. 1999, ApJ, 524, 1030

Ogilvie, G. I. 1999, MNRAS, 304, 557

Ogilvie, G. I., \& Dubus, G. 2001, MNRAS, 320, 485

Papaloizou, J. C., \& Pringle, J. E. 1983, MNRAS, 202, 1181

Papaloizou, J. C., \& Terquem, C. 1995, MNRAS, 274, 987

Pfeiffer, H., \& Lai, D. 2002, ApJ, in preparation

Pringle, J. E. 1996, MNRAS, 281, 857

Schaffer, L., \& Burns, J. A. 1992, Icarus, 96, 65

Shirakawa, A., \& Lai, D. 2002a, ApJ, 564, 361

Shirakawa, A., \& Lai, D. 2002b, ApJ, 565, 1134

Stella, L., \& Vietri, M. 1998, ApJ, 492, L59 